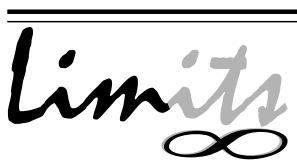

J. Math. and Its Appl.

ISSN : 1829-605X

Vol. 4, No. 1, May 2007, 1-8

\title{
The Application of The Steepest Gradient Descent for Control Design of Dubins Car for Tracking a Desired Path
}

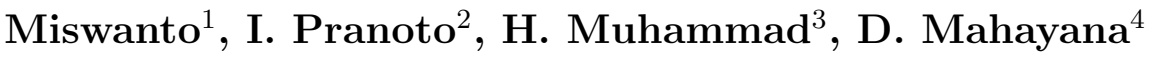 \\ ${ }^{1,2}$ Mathematics, \\ Miswanto@students.itb.ac.id, ranoto@dns.math.itb.ac.id \\ ${ }^{3}$ Aeronautics and Astronautics, \\ harmad@ae.itb.ac.id \\ ${ }^{4}$ Control System and Computer (STEI), \\ dmahayana@yahoo.com \\ Institut Teknologi Bandung, Indonesia
}

\begin{abstract}
In this paper, we consider the control design of the Dubins car system to track a desired path. We design the control of the Dubins car system using optimal control approach. The control of the Dubins car system is designed for tracking the desired path. Instead of the usual quadratic cost function, a special type of cost functional which includes a tracking error term will be considered. By this special cost functional, the minimum tracking error of path of the Dubins car toward a desired path using Pontryagin Maximum Principle is obtained. The analytical solution of the Hamiltonian system is difficult to obtain. So, a numerical solution with the steepest gradient descent method is proposed. The numerical results are given at the last section of this paper.
\end{abstract}

Keywords: Dubins Car System, Hamiltonian system, Gradient Descent, Numerical Simulation. 


\section{Introduction}

In recent years, there has been an increasing number of research on the subject of mobile robotics. This is because mobile robots are increasingly required in industry. For instance, to access difficult or dangerous area, such as space, military, or nuclear waste, unmanned mobile robots are a must.

The tracking problem is a challenging problem in mobile robotics. Some reseachers have discussed the control design of a mobile robot to track a desired path in [1], [2], [3], [5],[8]. In [1], the authors discuss the tracking control of mobile robots using integrator backstepping. Many mechanical systems with nonholonomic contraints can be locally or globally converted to the chained form under coordinate change. Jiang and Nijmeijer [2] study the tracking control problem of nonholonomic system in chained form. They derive semi global tracking controllers for general chained form systems by means of backstepping and they achieve global tracking results for some special cases. In [3], an adaptive tracking control problem is studied for a four wheel mobile robot. The authors propose a formulation for the adaptive tracking problem that meets the natural prerequisite such that it reduces to the state feedback tracking problem if the parameters are known. They derive a general methodology for solving their problem. In [5], Panteley et all study exponential tracking control of a mobile car using a cascaded approach. They show that the nonlinear controllers proposed in [1] can be simplified into linear controller for both the kinematic model and a simple dynamic model of the mobile robot. Their approach is based on cascaded system. In [8], the authors study a sufficient condition for the full state tracking stability of nonholonomic wheeled mobile robots by using the tracking control schemes based on the input output dynamics. They show that the tracking error internal dynamics and zero dynamics play a critical role of the full state tracking stability of such mobile robots. In [6], Tang et al. study optimal output tracking control (OOTC) problem for a class of bilinear systems with a quadratic performance index using a successive approximation approach (SAA). They develop a design process of the OOTC law based on the SAA for bilinear system. In [4], Miswanto et al. study the tracking problem of a swarm model with the presence of a leader by using the least square method. That model is a control system which consists of many agents and one agent has a role as a leader. The control of optimal motion of the leader is obtained by using the Pontryagin Maximum Principle. In particular, this control steers the leader to trace a desired path.

In this paper, the task is to steer the Dubin's car to trace a given path. The control of the Dubin's car system is obtained through the solution of the Hamiltonian system, using The Steepest Gradient Descent Method (Tjahjana [7]). In the next section, the formal problem formulation is described. In section 3, we 
show numerical simulations to illustrate our results.

\section{Problem Formulation}

In this section, we introduce the kinematic model of the Dubin's car system as shown in Figure 1.

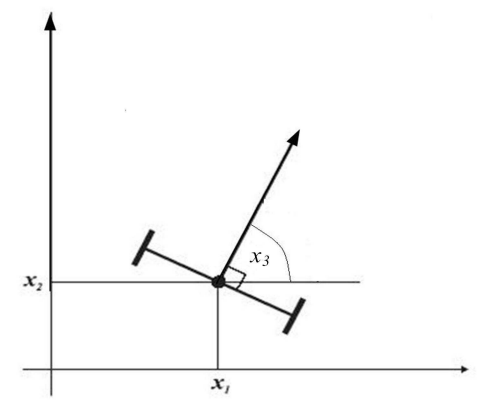

Figure 1: Dubin's Car System.

The kinematic model can be written as:

$$
\begin{aligned}
\dot{x_{1}} & =u_{1} \cos \left(x_{3}\right) \\
\dot{x_{2}} & =u_{1} \sin \left(x_{3}\right) \\
\dot{x_{3}} & =u_{2} \\
y & =\left[\begin{array}{lll}
x_{1} & x_{2} & x_{3}
\end{array}\right]^{T}
\end{aligned}
$$

where $\left(x_{1}, x_{2}\right)$ denote the position of the Dubin's car, $x_{3}$ is the orientation of the car, and $u_{1}, u_{2}$ are the linear and angular velocities, respectively. Here, $y$ is the output of the system. Thus, the output is the position and orientation of the car. In this paper, the desired path $\gamma$ that would be tracked by the Dubin's car is obtained using calculus variational method. That is, we consider the variational problem:

$$
\min _{\gamma} \int_{0}^{T} L(\ddot{\gamma}) d t,
$$

where $\gamma(0)=A, \gamma(T)=B, \dot{\gamma}(0)=C, \dot{\gamma}(T)=D$, and $A, B, C, D \in \mathbf{R}^{2}$ are given. The optimal solution of this variational calculus method is denoted by $\gamma(t)=$ 
$\left(\gamma_{1}(t), \gamma_{2}(t)\right)$

The original problem is translated to the following optimal control problem. We search for $\left(u_{1}, u_{2}\right)$ that makes the following functional minimized,

$$
J=\frac{1}{2} \int_{0}^{5}\left(\left(u_{1}\right)^{2}+\left(u_{2}\right)^{2}+\|e\|^{2}\right) d t .
$$

The term $\left(\left(u_{1}\right)^{2}+\left(u_{2}\right)^{2}\right)$ represents the total cost of the control used by the Dubin's car. The term $\|e\|^{2}$ represents the total error, where

$$
e(t)=\left[\begin{array}{ll}
x_{1}(t)-\gamma_{1}(t) & x_{2}(t)-\gamma_{2}(t)
\end{array}\right]^{T} .
$$

Here, $\left(x_{1}, x_{2}\right)$ is the state variable of the system (1). The error term in (3) is used to minimize the distance between the Dubin's car path and the desired path. Now, the Hamiltonian function of the system is $H=p_{1} u_{1} \cos \left(x_{3}\right)+p_{2} u_{1} \sin \left(x_{3}\right)+$ $p_{3} u_{2}+\frac{1}{2} p_{0}\left(\left(u_{1}\right)^{2}+\left(u_{2}\right)^{2}+\|e\|^{2}\right)$. Using this function, we build the Hamiltonian system:

$$
\begin{aligned}
& \frac{\partial H}{\partial p_{1}}=\dot{x_{1}}=u_{1} \cos \left(x_{3}\right) \\
& \frac{\partial H}{\partial p_{2}}=\dot{x_{2}}=u_{1} \sin \left(x_{3}\right) \\
& \frac{\partial H}{\partial p_{3}}=\dot{x_{3}}=u_{2} \\
& \frac{\partial H}{\partial x_{1}}=-\dot{p_{1}}=p_{0}\left(x_{1}(t)-\gamma_{1}(t)\right) \\
& \frac{\partial H}{\partial x_{2}}=-\dot{p_{2}}=p_{0}\left(x_{2}(t)-\gamma_{2}(t)\right) \\
& \frac{\partial H}{\partial x_{3}}=-\dot{p_{3}}=-p_{1} u_{1} \sin \left(x_{3}\right)+p_{2} u_{1} \cos \left(x_{3}\right) .
\end{aligned}
$$

By the Pontryagin Maximum principle, the value of $H$ must be optimized with respect to the control $\left(u_{1}, u_{2}\right)$. Thus,

$$
\begin{aligned}
& \frac{\partial H}{\partial u_{1}}=p_{1} \cos \left(x_{3}\right)+p_{2} \sin \left(x_{3}\right)+p_{0} u_{1}=0 \\
& \frac{\partial H}{\partial u_{2}}=p_{3}+p_{0} u_{2}=0,
\end{aligned}
$$

since $p_{0}$ must be constant and negative, without loss of generality, we let $p_{0}=-1$. Thus, we obtain the control

$$
u_{1}=p_{1} \cos \left(x_{3}\right)+p_{2} \sin \left(x_{3}\right)
$$




$$
u_{2}=p_{3}
$$

Then, this control is substituted in (5). Thus, we obtain a system of differential equations:

$$
\begin{aligned}
& \dot{x_{1}}=p_{1} \cos ^{2}\left(x_{3}\right)+p_{2} \sin \left(x_{3}\right) \cos \left(x_{3}\right) \\
& \dot{x_{2}}=p_{1} \sin \left(x_{3}\right) \cos \left(x_{3}\right)+p_{2} \sin ^{2}\left(x_{3}\right) \\
& \dot{x_{3}}=p_{3} \\
& \dot{p_{1}}=\left(x_{1}(t)-\gamma_{1}(t)\right) \\
& \dot{p_{2}}=\left(x_{2}(t)-\gamma_{2}(t)\right) \\
& \dot{p_{3}}=\left(p_{1}^{2}-p_{2}^{2}\right) \sin \left(x_{3}\right) \cos \left(x_{3}\right)+p_{1} p_{2}\left(\sin ^{2}\left(x_{3}\right)-\cos ^{2}\left(x_{3}\right)\right) .
\end{aligned}
$$

Initial and final conditions of the state variables $\left(x_{i} ; i=1,2,3\right)$ are known. However, the costate equations $\left(p_{i} ; i=1,2,3\right)$ does not have initial condition. So, the solution of the system of differential equation is difficult to obtain. This paper proposes the steepest gradient descent method. This method is used to approximate the initial condition of the costate variables in the system of differential equation. First, the initial value of state variables are given by $x_{i}(0)=x_{i 0} ; i=1,2,3$ and the initial value of costate variables are arbitrarily guessed by $p_{i}(0)=q_{i 0} ; i=1,2,3$. The values are used to solve the system of differential equation. Next, we calculate $F\left(q_{10}, q_{20}, q_{30}\right)=\sum_{i=1}^{3}\left\|x_{i}(T)-x_{i T}\right\|^{2}$, where $x_{i}(T)$ is obtained from the solution of the system of differential equation and is the final conditions of the state variables. Afterwards, we determine the value of the new $\left(q_{10}, q_{20}, q_{30}\right)$ by using the steepest gradient descent method, such as in Tjahjana [7]. The value is used to make the new $F\left(q_{10}, q_{20}, q_{30}\right)$ less than the old $F\left(q_{10}, q_{20}, q_{30}\right)$. The process is done repeatedly until the value of $F\left(q_{10}, q_{20}, q_{30}\right)$ is small enough.

\section{Numerical Simulation}

In this section, numerical simulations to illustrate the proposed method are reported. The initial and boundary conditions of the desired path are given by $\gamma(0)=(1,2), \gamma(5)=(5,4), \dot{\gamma}(0)=(1,0)$, and $\dot{\gamma}(5)=(1,0)$. A desired path obtained by the calculus variational method is the following parametric curve:

$$
\begin{aligned}
& \gamma_{1}(t)=\frac{2}{125} t^{3}-\frac{3}{25} t^{2}+t+1 \\
& \gamma_{2}(t)=-\frac{4}{125} t^{3}+\frac{6}{25} t^{2}+2
\end{aligned}
$$

The Dubin's car is expected to maneuver tracing this path as close as possible. The initial and final conditions of the Dubin's car system are given by 
$\left(x_{1}(0), x_{2}(0), x_{3}(0)\right)=(1,2,0)$, and $\left(x_{1}(5), x_{2}(5), x_{3}(5)\right)=(5,4,0)$, respectively. The desired path in equation (7) is substituted in (6). Thus, we obtain a system of differential equations:

$$
\begin{aligned}
\dot{x_{1}} & =p_{1} \cos ^{2}\left(x_{3}\right)+p_{2} \sin \left(x_{3}\right) \cos \left(x_{3}\right) \\
\dot{x_{2}} & =p_{1} \sin \left(x_{3}\right) \cos \left(x_{3}\right)+p_{2} \sin ^{2}\left(x_{3}\right) \\
\dot{x_{3}} & =p_{3} \\
\dot{p_{1}} & =\left(x_{1}(t)-\frac{2}{125} t^{3}+\frac{3}{25} t^{2}-t-1\right) \\
\dot{p_{2}} & =\left(x_{2}(t)+\frac{4}{125} t^{3}-\frac{6}{25} t^{2}-2\right) \\
\dot{p_{3}} & =\left(p_{1}^{2}-p_{2}^{2}\right) \sin \left(x_{3}\right) \cos \left(x_{3}\right)+p_{1} p_{2}\left(\sin ^{2}\left(x_{3}\right)-\cos ^{2}\left(x_{3}\right)\right)
\end{aligned}
$$

The numerical solution of the above equation utilizes the steepest gradient descent method. Fig. 2 shows the path of the Dubin's car tracing the desired path by using the method. Table 1 shows the error.

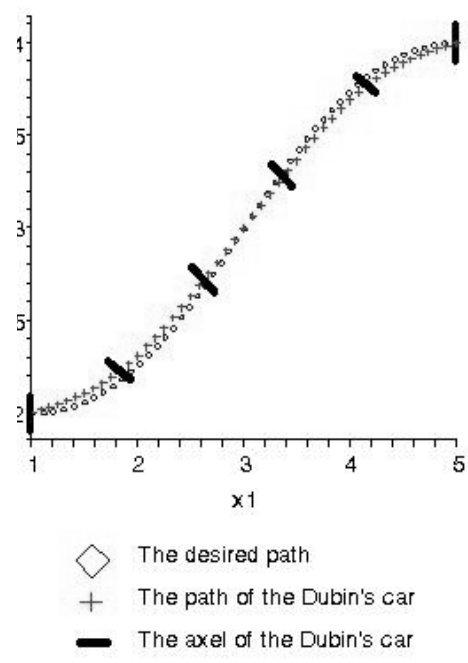

Figure 2: The trajectories of desired path and the optimal trajectory of the car.

\section{Future Works}

From the numerical simulation results above, it can be seen that the tracking error of the path of the Dubin's car tracing a desired path is sufficienty small. In 
Tabel 1: Error

\begin{tabular}{|c|c|c|c|c|c|}
\hline Time $(\mathrm{t})$ & Error & Time $(\mathrm{t})$ & Error & Time $(\mathrm{t})$ & Error \\
\hline 0 & $5.95 \mathrm{E}-07$ & 1.75 & $2.05 \mathrm{E}-07$ & 3.5 & $3.11 \mathrm{E}-07$ \\
\hline 0.25 & $8.19 \mathrm{E}-07$ & 2 & $1.02 \mathrm{E}-07$ & 3.75 & $3.87 \mathrm{E}-07$ \\
\hline 0.5 & $2.30 \mathrm{E}-07$ & 2.25 & $2.70 \mathrm{E}-07$ & 4 & $4.06 \mathrm{E}-07$ \\
\hline 0.75 & $3.52 \mathrm{E}-07$ & 2.5 & $3.45 \mathrm{E}-07$ & 4.25 & $3.52 \mathrm{E}-07$ \\
\hline 1 & $4.07 \mathrm{E}-07$ & 2.75 & $2.69 \mathrm{E}-07$ & 4.5 & $2.30 \mathrm{E}-07$ \\
\hline 1.25 & $3.88 \mathrm{E}-07$ & 3 & $1.01 \mathrm{E}-07$ & 4.75 & $8.81 \mathrm{E}-07$ \\
\hline 1.5 & $3.11 \mathrm{E}-07$ & 3.25 & $2.05 \mathrm{E}-07$ & 5 & $5.98 \mathrm{E}-07$ \\
\hline
\end{tabular}

the future works, we will discuss the movement control of swarm system consisting of several Dubin's cars with a specific geometry formation.

\section{Pustaka}

[1] Z-P. Jiang and H. Nijmeijer, 1997, Tracking Control of Mobile Robots:a Case Study in Backstepping, Automatica, vol. 33, no. 7, pp. 1393-1399.

[2] Z-P. Jiang and H. Nijmeijer, 1999b, A Recursive Technique for Tracking Control of Nonholonomic Systems in Chained Form, IEEE Transaction on Automatic Control, vol. 44, no. 2, pp. 256-279.

[3] E. Lefeber And H. Nijmeijer, December 1999, Adaptive Tracking Control of Nonholonomic Systems: an example, Proceedings of the 38th Conference on Decision and Control, Phoenix, Arizona USA.

[4] Miswanto, I. Pranoto, and H. Muhammad, 2006, A Model of Swarm Movement with The Presence of A Leader, Proceeding, International Conference on Mathematics and Natural Sciences, pp. 740-742, ITB, Bandung.

[5] E. Panteley, E. Lefeber, A. Loria, and H. Nijmeijer, September 1998, Exponential Tracking Control of a Mobile Car Using a Cascaded Approach, Proceeding of the IFAC Workshop on Motion Control, pp. 221-226, Grenoble France.

[6] G.Y. Tang, Y.D. Zhao and Hui Ma, 2006, Optimal Output Tracking Control for Bilinear Systems, Transactions of the Institute of Measurement and Control 28, 4, pp. 387-397.

[7] H. Tjahjana, I. Pranoto, H. Muhammad, and J. Naibourho, 2007, On the optimal control Computation of linear system, preprint. 
[8] D. Wang and G. Xu, June 2000, Full State Tracking and Internal Dynamics of Nonholonomic Wheeled Mobile Robots, Proceedings of the American Control Conference, pp. 3274-3278, Chicago, Illinois. 